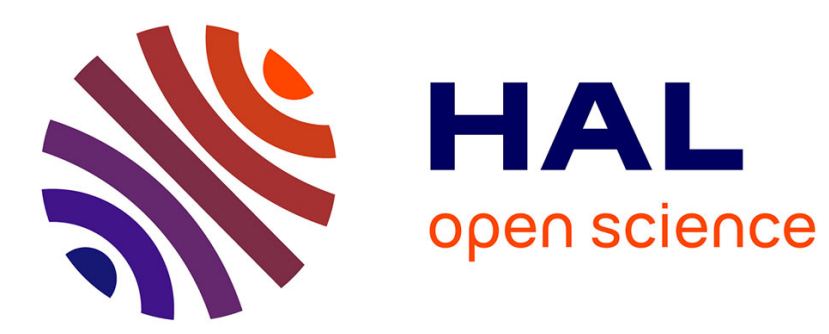

\title{
L'usage de la messagerie électronique: une méta-analyse des travaux francophones sur la période 2000-2008
}

\author{
Wadi Tahri, Bernard Fallery
}

\section{To cite this version:}

Wadi Tahri, Bernard Fallery. L'usage de la messagerie électronique: une méta-analyse des travaux francophones sur la période 2000-2008. Revue management \& avenir, 2010, 34, pp.184-200. 10.3917/mav.034.0183 . hal-00773676

\section{HAL Id: hal-00773676 https://hal.science/hal-00773676}

Submitted on 14 Jan 2013

HAL is a multi-disciplinary open access archive for the deposit and dissemination of scientific research documents, whether they are published or not. The documents may come from teaching and research institutions in France or abroad, or from public or private research centers.
L'archive ouverte pluridisciplinaire HAL, est destinée au dépôt et à la diffusion de documents scientifiques de niveau recherche, publiés ou non, émanant des établissements d'enseignement et de recherche français ou étrangers, des laboratoires publics ou privés. 


\section{L'usage de la messagerie électronique : une méta-analyse des travaux francophones sur la période 2000-2008}

\author{
Wadi TAHRI \\ CREGOR, \\ Université Montpellier 2 \\ Place Eugène Bataillon, Bât. 19, \\ 34095 Montpellier Cedex 5 \\ E-mail : wadi.tahri@iutbeziers.fr \\ Tél. : 06-07-60-06-37 \\ Fax : 04-67-14-42-20
}

\author{
Bernard Fallery \\ CREGOR,
}

\section{Université Montpellier 2}

Place Eugène Bataillon, Bât. 19, 34095 Montpellier Cedex 5

E-mail : bernard.fallery@univ-montp2.fr

Tél. : 04-67-14-42-21

Fax : 04-67-14-42-20

\section{ü Résumé}

La messagerie électronique s'est rapidement développée dans les organisations aux cours de ces dix dernières années, par sa facilité d'utilisation et son utilité perçue. Pourtant, Weber publie en 2004 un article dans le MIS Quaterly dans lequel il soulève : " Curieusement, les recherches sur la messagerie électronique sont peu significatives en systèmes d'information ». Il invite alors les chercheurs à publier davantage sur «l'e-mail ». Cet article présente une méta-analyse des travaux de recherche francophones sur la période 2000-2008. Nous étudions l'usage de la messagerie électronique dans les organisations à l'aide d'une analyse statistique des données textuelles. Dans un premier temps, nous expliquons l'originalité de cette démarche et le traitement des données. Dans un deuxième temps, nous présentons les résultats et les profils de classes obtenus par Alceste : quatre classes différentes, correspondant à quatre grands sujets de recherche : «Temps et productivité », « Choix des médias et productivité »; «Utilisation/satisfaction », « Interface homme/machine ». Enfin, nous discutons des résultats, de la validité et des limites de cette démarche. Ceci ouvre des pistes de réflexions et des voies de recherches futures.

\section{Mots clefs :}

Usage, appropriation, méta-analyse, TIC, messagerie électronique. 


\section{INTRODUCTION}

Les innovations technologiques ont apporté des évolutions profondes au travail humain. Par la lecture de différents travaux (Davis et al. 1989, 2003; Orlikowski et Baroudi 1992; DeSanctis et Poole, 1994 ; Weick, 1995 ; DeVaujany, 2000 ; Reix, 2002...) sur les usages des TIC, ces évolutions provoquent des effets très différents selon les métiers et les individus. Subir le changement, accepter de découvrir une technologie, comprendre le vocabulaire utilisé, apprendre et surmonter la fracture numérique, les appropriations constituent aujourd'hui une question centrale en systèmes d'information.

Les processus de communication sont au cœur du fonctionnement de l'organisation. Néanmoins, leur analyse en révèle la complexité, comme l'évoque l'approche systémique (Wtazlawick et al., 1972 ; Le Moigne, 1990 ; Simon, 1991 ; De Rosnay, 1975), soulignant que tout comportement est adopté en interaction avec autrui et que tout équilibre est en interaction avec l'environnement. Il est donc clair que la communication ne peut se réduire à la simple transmission d'un message plus ou moins codé (Reix, 2004). Si l'implantation de la messagerie électronique est arrivée aujourd'hui à la phase de maturité, les résultats des travaux de recherches sont encore contradictoires (Limayem et al., 1997; Raymond et Bergeron, 1996 ; D'Iribarne et Gadille, 2000 ; Jouêt 2000 ; Raymond 2002) et relèvent des situations extrêmement contrastées (Van den Hooff et al., 2005), voire même dans le même contexte (Boukef et Charki, 2008).

A priori, les recherches dans notre discipline sur la messagerie électronique ${ }^{1}$ se réduisent très souvent à l'étude des facteurs d'adoption à la lumière du TAM (Davis, 1989). La métaanalyse de Yousafzai et al. (2007) ne contredira pas nos propos puisqu'elle estime que c'est l'un des modèles les plus testés. D'autres recherches comparent les moyens de communication à la lumière de la théorie de la richesse des média (Daft et Lengel, 1986 ; Kalika et al., 2007). Or, la question aujourd'hui ne se pose pas de savoir s'il faut ou non utiliser la messagerie électronique (surtout quand l'utilisation est rendue quasiment banale et obligatoire), il convient plutôt de s'interroger sur la capacité de l'individu à choisir parmi les différents canaux de communication disponibles le "bon" moyen pour travailler "efficacement", ainsi qu'aux pratiques des utilisateurs qui traitent et échangent les informations dans une perspective interactionniste (qui s'oppose au déterminisme) pour comprendre la complexité réelle de l'usage de la technologie dans les contextes de la communication organisationnelle.

Dans ce cadre d'analyse, nous centrons notre choix d'étudier le média sur la période 20002008. Ce choix est d'abord justifié par la diffusion généralisée de l'outil à tous les secteurs d'activité et à tous les membres de l'organisation. En 2002, un peu moins de la moitié des employés utilisent la messagerie électronique ${ }^{2}$. En 2007, l'outil devient pour la première fois l'outil principal de travail et remplace les moyens traditionnels de communication existants

\footnotetext{
${ }^{1}$ Nous utiliserons indifféremment les termes messagerie électronique, courrier électronique et e-mail dans cet article, compte tenu de leur confusion dans la recherche francophone.

${ }^{2}$ Source : Sessi, Scees et Insee, enquête TIC et commerce électronique, fin 2002.
} 
comme le fax et le téléphone ${ }^{3}$. La messagerie a permis aux employés de passer au monde du numérique : les zones de collaboration s'étendent, la distance et le temps ne constituent plus aujourd'hui des freins pour la productivité. De plus, aujourd'hui l'e-mail a pris de plus en plus d'importance avec son extension dans les technologies mobiles (téléphone, PDA), avec la généralisation du haut débit et avec sa reconnaissance juridique comme une preuve légale dans l'entreprise.

\section{Problématique :}

Si l'implantation de la messagerie électronique est arrivée aujourd'hui à la phase de maturité, les résultats des travaux de recherches sont encore contradictoires (Limayem et al., 1997 ; Raymond et Bergeron, 1996 ; D'Iribarne et Gadille, 2000 ; Jouêt 2000 ; Raymond 2002) et relèvent des situations extrêmement contrastées (Van den Hooff et al., 2005).

Limayem et al. (1997) comparent les mesures objectives et subjectives de la messagerie électronique à travers le modèle de Triandis. Van den Hooff et al. (2005) proposent une métaanalyse de 17 articles pour comparer les différentes approches théoriques expliquant son usage. A l'instar de ces recherches, nous proposons une méta-analyse des travaux francophones ayant traité cette technologie au cours de la période 2000-2008 et nous décrivons l'état d'avancement des recherches sur le sujet. Une première lecture de résultats souvent contradictoires semble révéler deux courants : celui du déterminisme technologique et celui du déterminisme organisationnel. Ceci justifie alors une analyse statistique des études sur le sujet pour tenter de répondre aux questions suivantes : De quoi parle-t-on exactement dans la littérature? Quelles sont les méthodes de validation ? Quels sont les résultats obtenus?

\section{Objectifs de la recherche :}

Weber publie en 2004 un article dans le MIS Quaterly dans lequel il soulève : "Curieusement, les recherches sur la messagerie électronique sont peu significatives en systèmes d'information ". Il semble à travers ce propos que l'état de l'art des travaux existants ne permet pas encore de résoudre les problèmes liés à la messagerie électronique et invite alors les chercheurs à publier davantage sur le sujet. En ce sens, il serait réducteur d'étudier les avantages qu'apporte une technologie, Orlikowski (2003) relativise les investissements en technologie de l'information et suggère à s'interroger davantage sur leurs réels usages et appropriations : "En négligeant l'utilisation de la technologie, nous oublions que celle-ci n'a ni valeur, ni sens, ni conséquences par elle-même : c'est la pratique qui décide de tout cela. Omettre ce fait, c'est ouvrir la porte à trois suppositions simplistes : 1) si le personnel dispose de la technologie, il l'utilisera forcément ; 2) il l'utilisera dans le sens où elle a été conçue ; 3) cette utilisation produira les résultats attendus ».

Par ailleurs, la demande faite par l'entreprise qui a hébergé notre travail de recherche en témoigne des difficultés nouvelles que rencontrent les employés dans leurs activités professionnelles et relations personnelles.

\footnotetext{
${ }^{3}$ Selon l'étude réalisée par le cabinet Datamonitor dans 524 entreprises de treize pays, 100\% des personnes interrogées utilisent l'e-mail, $80 \%$ le téléphone, $76 \%$ le téléphone mobile et $66 \%$ la messagerie instantanée.
} 
Nous cherchons non seulement à connaître l'apport de la recherche francophone sur le sujet, mais également à déterminer les différentiations d'usage, d'une part, et les processus d'appropriation qui relève de la construction de sens (Weick, 1995), d'autre part.

Dans le premier point, nous présentons la méta-analyse des articles francophones étudiant l'usage de la messagerie électronique, en expliquant l'originalité de notre démarche et le traitement des données. Le deuxième point présente les résultats et les profils des classes obtenus à l'aide du logiciel Alceste. Dans le troisième point nous discutons des résultats en comparant avec une méta-analyse des travaux anglophones, ainsi que la validité et des limites de cette démarche.

\section{LA META-ANALYSE}

Malgré l'ampleur des travaux de recherche sur la communication électronique, de nombreux problèmes conceptuels et de validité des instruments doivent encore être surmontés.

"Meta-analysis refers to the analysis of analyses...the statistical analysis of a large collection of analysis results from individual studies for the purpose of integrating the findings" (Glass, 1976).

\subsection{Démarche de la méta-analyse}

La méta-analyse est une démarche, plus qu'une simple technique, qui a pour but de combiner les résultats de plusieurs études pour en faire une synthèse reproductible et quantifiée. Glass (1976) a élaboré la démarche des méta-analyses dans le domaine des sciences sociales et de l'éducation. Dans les années suivantes, cette technique fut largement employée en médecine, car elle permet de conjuguer les essais obtenus aux travers de plusieurs dizaines d'études et de généraliser les résultats. La méta-analyse est également un instrument précieux pour les sciences de gestion (Commeiras et Fournier, 2008). Elle devrait jouer un rôle clé dans la validation des travaux de recherches en systèmes d'information où les résultats varient souvent d'une étude à l'autre.

Comme pour toute méthodologie, il est important d'expliquer les différentes phases permettant d'aboutir aux résultats. Tout d'abord, nous avons procédé à une démarche de sélection des travaux pour la méta-analyse sur le sujet de la messagerie électronique à travers une série de questions: Que cherche-t-on à démontrer? Quelles sont les hypothèses ou propositions ? S'agit-il d'une étude empirique ou exploratoire ? Quantitative ou qualitative ? Quels sont les résultats? Ensuite, nous avons sélectionné les études les plus significatives traitant spécifiquement la thématique (communications ou publications entre 2000 et 2008). Le but de cette étape est de définir l'information essentielle contenue dans les articles présélectionnés pour en tirer une conclusion dotée d'une signification statistique plus grande. Enfin, après vérification de la similitude des études, la démarche nous a permis de ne retenir que vingt articles (Tableau 1). 
Introduction de la messagerie et des

forums dans l'entreprise : quels

Bachelet C., Caron-Fasan

impacts pour l'organisation? Une

M.L. (2000)

approche exploratoire

L'utilisation de la messagerie

Autissier D. (2000)

électronique dans une

multinationale : l'émergence d'un

nouveau média

\begin{tabular}{|c|c|}
\hline $\begin{array}{l}\text { Grandeurs et limites de la } \\
\text { communication électronique : } \\
\text { analyse d'un cas de projet de } \\
\text { recherche marketing. }\end{array}$ & $\begin{array}{l}\text { Guilloux V., Gauzente C. et } \\
\text { Kalika M. (2000) }\end{array}$ \\
\hline $\begin{array}{l}\text { Limites structurels et culturelles à } \\
\text { l'usage de la messagerie électronique } \\
\text { dans les banques }\end{array}$ & Rowe F., Monod E. (2000) \\
\hline $\begin{array}{l}\text { Trois formes différenciées d'usage de } \\
\text { la messagerie électronique au sein } \\
\text { d'une organisation }\end{array}$ & Saintive B. (2000) \\
\hline $\begin{array}{l}\text { La messagerie électronique, facteur } \\
\text { de changement dans l'organisation? } \\
\text { Implication sur la décision }\end{array}$ & $\begin{array}{l}\text { Bachelet C., Moscarola J. } \\
(2001)\end{array}$ \\
\hline $\begin{array}{l}\text { La messagerie électronique dans la } \\
\text { communication : usages, contextes, } \\
\text { et satisfaction }\end{array}$ & $\begin{array}{l}\text { Bachelet C., Moscarola J. } \\
(2002)\end{array}$ \\
\hline $\begin{array}{l}\text { L'impact d'Internet sur le } \\
\text { fonctionnement des moyennes et } \\
\text { grandes entreprises industrielles }\end{array}$ & Barry P., Diop H. (2002) \\
\hline $\begin{array}{l}\text { Facteurs déterminants de l'utilisation } \\
\text { du courrier électronique }\end{array}$ & $\begin{array}{l}\text { Boukef N., Kalika M. } \\
(2002)\end{array}$ \\
\hline $\begin{array}{l}\text { Complémentarité et substitution du } \\
\text { téléphone et du courrier } \\
\text { électronique : une approche par } \\
\text { typologie prédictive }\end{array}$ & $\begin{array}{l}\text { Cucchi A., Cucchi C. } \\
\text { (2003) }\end{array}$ \\
\hline $\begin{array}{l}\text { Le succès de l'utilisation de la } \\
\text { messagerie électronique - étude de } \\
\text { ses déterminants au sein d'une unité } \\
\text { de production aéronautique }\end{array}$ & Baile S., Lefievre V. (2003) \\
\hline
\end{tabular}

Messagerie électronique synchrone

Gléonnec M. (2004)

et structuration du lien social en entreprise

Représentation graphique de la communication organisationnelle Cucchi C. (2004) par les réseaux sociaux ; exemple des échanges électroniques

Pourquoi et comment évaluer l'Interaction Homme/Machine? Le

Baile S. (2004)

cas de la messagerie électronique dans les processus d'apprentissage

La théorie du millefeuille. De la non-substitution entre

Kalika M., Boukef N. et 


\begin{tabular}{ll}
\hline communications électroniques et face à face & Isaac H. (2005) \\
\hline $\begin{array}{l}\text { Communication numérique et continuité des genres : l'exemple du } \\
\text { courrier électronique }\end{array}$ & $\begin{array}{l}\text { Labbe H., Marcoccia M. } \\
\text { (2005) }\end{array}$ \\
\hline $\begin{array}{l}\text { La messagerie instantanée en entreprise : Accélérateur ou frein à la } \\
\text { productivité ? }\end{array}$ & $\begin{array}{l}\text { Cudicio R., Proulx S. } \\
\text { (2006) }\end{array}$ \\
\hline $\begin{array}{l}\text { Influence de l'utilité perçue et de la facilité d'usage sur l'utilisation } \\
\text { de la messagerie électronique: application au contexte tunisien }\end{array}$ & $\begin{array}{l}\text { Daoud H., Hassairi A.F, } \\
\text { Triki M. (2007) }\end{array}$ \\
\hline $\begin{array}{l}\text { L'impact des TIC sur la productivité : la dimension oubliée de la } \\
\text { structuration des usages ? Le cas de l'utilisation de l'email dans les } \\
\text { secteurs de service }\end{array}$ & Rolland S., Tran S. (2007) \\
\hline
\end{tabular}

La messagerie électronique dans les multinationales : sources de Tahri W. (2008)

productivité ou de conflits ? Vers une approche normative

\section{Tableau 1 : Les articles sélectionnés}

Cet échantillon est justifié par la disponibilité de données de qualité. Nous nous sommes assurés de prendre des recherches diverses (différents contextes), mais similaires (traitant uniquement de la messagerie électronique). Il s'agit en effet d'éviter une sélection biaisée, et pour cela d'analyser des études comparables. C'est une analyse de données textuelles via le logiciel Alceste que nous avons adoptée pour effectuer la méta-analyse.

\subsection{Traitement des données par le logiciel Alceste}

Les questions que nous nous sommes posées à ce stade étaient: Quelle approche pour l'analyse de données textuelles doit-on adopter (lexicale, linguistique, cognitive, thématique) ? Quel logiciel pour le traitement (Nvivo, Sphinx, Alceste, SpadT...) ? Il s'agit "d'augmenter la validité des analyses de contenu " classiques » et de laisser surgir des notions nouvelles tout en proposant des garde-fous à des interprétations trop projectives » (Fallery et Rodhain, 2007).

Nous nous sommes assurés par le recours à l'outil lexical Alceste d'une certaine objectivité du traitement automatique des articles sélectionnés. En effet, l'utilisation d'un logiciel est nécessaire pour effectuer une analyse lexicale et extraire les structures (phrases+mots) les plus signifiantes. Les traitements ne sont pas influencés par le chercheur, car les mécanismes sur lesquels repose le logiciel sont indépendants de l'analyste.

2.2.1. Le prétraitement: Nous avons regroupé et revu l'intégralité du corpus pour détecter toute éventuelle erreur susceptible de biaiser les résultats. Ainsi, nous avons effectué les opérations suivantes sur le corpus, afin de minimiser les risques de «non traitement» des mots :

Suppression des tableaux, schémas,...;

Suppression des dates et des noms propres des auteurs ;

Suppression des caractères spéciaux (guillemets, pourcentages,...);

Suppression des entêtes et pieds de pages (pour éviter la redondance de mots) ; 
2.2.2. Le traitement: La méthode de l'analyse de données textuelles par Alceste ${ }^{4}$ a été adoptée pour définir de manière quantitative les caractéristiques de notre cadre de recherche. Des variables signalétiques «mots étoilés » ont été ajoutées pour chaque article, décrivant le type de la recherche (empirique, non empirique), l'objet étudié, la méthodologie et le type d'enquête (questionnaires, entretiens, étude de cas).

Alceste permet alors de dégager du corpus étudié les principaux « mondes lexicaux » en présence. Après lemmatisation (le remplacement d'une forme textuelle par sa forme réduite standardisée dans un dictionnaire), le logiciel découpe le texte en unités de sens, avec deux phases principales:

Phase 1 : découpage du texte en Unités de Contexte (UCI, UCE)

Phase 2 : Classification descendante hiérarchique (CDH)

\section{Les résultats de la méta-analyse}

A partir des 226 pages de texte du corpus, nous synthétisons ici les résultats par des présentations graphiques et une analyse de chacune des classes obtenues.

3.1. Présentations graphiques : Grâce à la méthode décrite ci-dessus, Alceste repère les similitudes les plus fortes et en extrait des classes. Pour chacune des classes, nous avons obtenu les mots et les phrases les plus significatifs, les segments répétés, les concordances des mots les plus caractéristiques.

Après la mise en forme des recherches sélectionnées, le texte analysé est constitué de 99008 mots et comporte 2676 U.C.E. Parmi celles-ci, 2055 ont été classées soit 76,79\% du total. Ce résultat met en évidence la fiabilité de l'analyse (qualité du corpus) dans le graphique suivant (figure 1) :
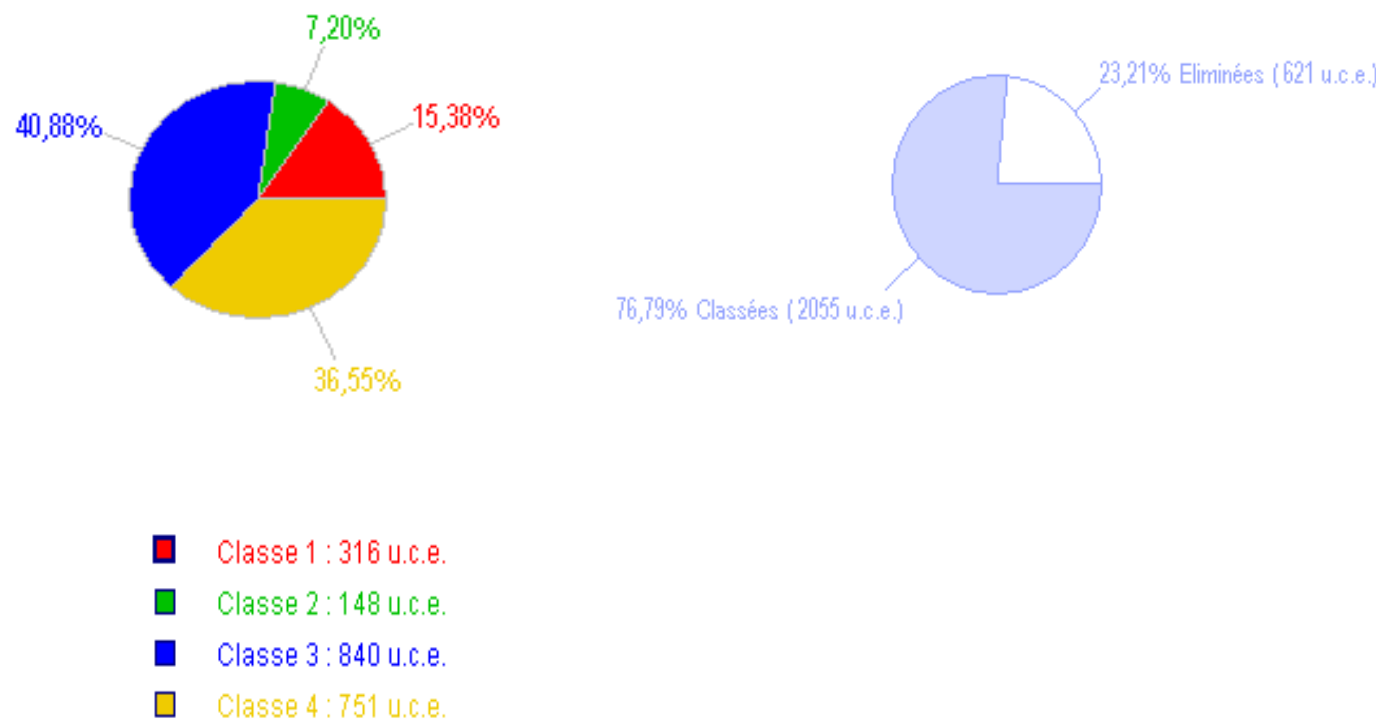

Figure 1 : Première classification des UCE dans le corpus

\footnotetext{
${ }^{4}$ Crée par Max Reinert (CNRS) en 1990, l'analyse s'est faite avec la version 4.7
} 
Dans un premier temps, il ressort que les classes 3 et 4 sont les plus caractérisées : 1591 des U.C.E. classées, soit $78 \%$. Les classes 1 et 2 ne représentent que $28 \%$ des $\mathrm{UCE}_{\text {rlacce }} 2$ (respectivement $15,38 \%$ et $7,2 \%$ ).

Dans un deuxième temps, l'analyse factorielle des correspondances (AFC) présentée dans la figure 2 montre une certaine similitude des études francophones traitant la messagerie électronique : la répartition des concepts est assez concentrée, les 3 classes principales se rejoignant au centre de la projection. Ceci est sans doute lié aux conditions de sélection des articles traitant les mêmes sujets.

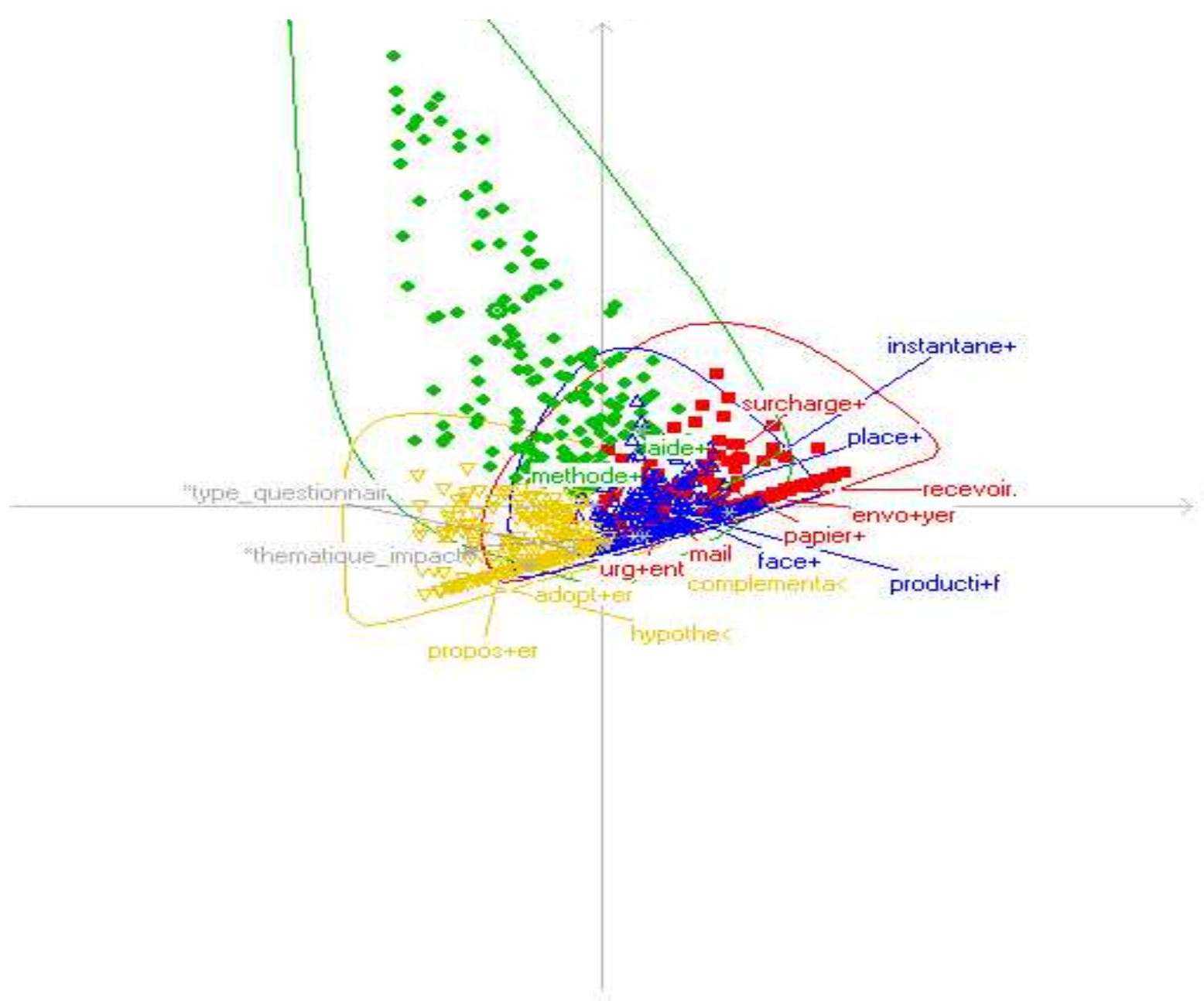

Classe 4

Classe 1

rlacse 2

Figure 2 : Analyse factorielle des correspondances

3.2. Profils des classes : Chaque classe obtenue constitue un concept particulier sur l'étude des usages de la messagerie électronique dans les organisations. La prise en compte du lexique utilisé nous a conduit à nommer les classes proposées par le logiciel. Ainsi, nous les analyserons dans un ordre décroissant du nombre d'U.C.E. (Figure 3). 


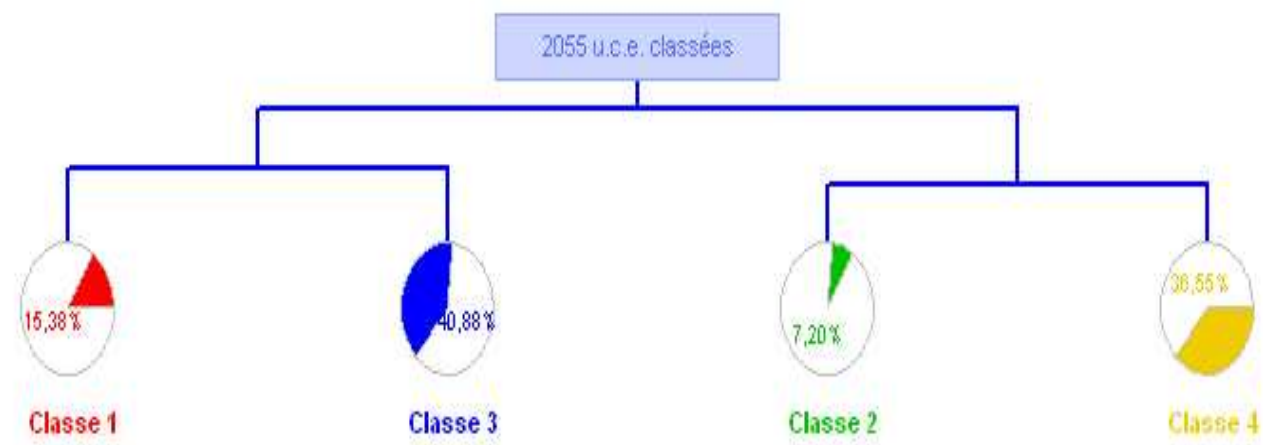

Figure 3 : Dendrogramme des classes

La classe 3 (contenant $41 \%$ des U.C.E) peut être intitulée «Le choix des médias et la productivité ». Elle regroupe un vocabulaire formé de noms, d'adjectifs et de peu de verbes. Les mots plus caractéristiques de cette classe sont présentés ci-dessous, chaque nombre correspond à l'indicateur $\mathrm{Khi}^{2}$ mesurant la significativité du mot dans la classe :

\begin{tabular}{l}
$\begin{array}{l}\text { Productif(97), reunion }+(62), \quad \text { chat }+(56), \quad \text { communic }<(54), \quad \text { MI(48), } \\
\text { instantane }+(38), \\
\text { forum }+(24) \ldots\end{array}$ \\
\hline
\end{tabular}

Si nous divisons cette classe en deux sous groupes par une analyse hiérarchique ascendante (CHA), nous obtenons les mots suivants :

- Sous-classe 3.1 : productif - réunion - communication - entreprise - face - forum présentent un Khi-2 très élevé dans la première sous-classe

- Sous-classe 3.2 : usager - privé - professionnel - écrit - relationnel - chat-réseau dans la deuxième sous-classe

Une phase de relecture directe dans le corpus des U.C.E. les plus caractéristiques de cette classe nous permet alors de constater qu'une grande majorité des travaux s'interroge sur le choix et la place de la messagerie électronique par rapport aux autres moyens de communication (sous-classe 3.1). Un deuxième grand débat fait apparaître la problématique vie professionnelle/ vie personnelle dans le cadre de la productivité et de l'usage des médias (sous-classe 3.2).

L'association de ces analyses en classes et de la relecture directe dans les articles des différentes relations entre variables nous permet donc d'avancer la proposition suivante :

Résultat A : La productivité associée à la messagerie électronique est, dans la plupart des travaux, liée au problème du choix des médias (messagerie, réunions, écrits, Chat...). 
La classe 4, que nous pouvons appeler "Utilisation/Satisfaction », illustre bien les variables de la théorie TAM (technology acceptance model) de Davis (1989) et du modèle de Delone et Mclean (2003) :

Varia+ble(223), Utilisa+tion(156), satisfaction+(141), influenc+er(132), hypothe $<(126)$, media+(102), modele+(93), explica<(82), theori<(80), resultat $+(69)$, riche+(66), percepti<(58), percevoir(53), age $+(50)$, usage $+(47)$, reati $+f(45) \ldots$

En effet, cette classe est centrée sur Utilisation et Satisfaction et elle souligne la méthodologie utilisée dans ces études. Nous pouvons la diviser également en deux sous classes :

- Dans la première sous classe 4.1, nous constatons dans l'ordre les items : usage, acceptation, satisfaction, perception, adoption.

- Dans la deuxième sous classe 4.2, les items : utilisation, variables (age, sexe,...), contexte, positif, effet... qui font référence aux variables les plus utilisées.

Le contenu de cette classe est relatif aux hypothèses étudiées. La relecture du corpus pour cette classe montre que les chercheurs francophones analysent ici l'influence des différentes variables sur les usages des médias. De ce fait, ils étudient les effets positif/négatif des variables individuelles et organisationnelles sur l'utilisation du média et la relation existante entre utilisation, satisfaction, acceptation et appropriation de la messagerie électronique.

Il apparaît donc que :

\section{Résultat B : La grande majorité des travaux sur la messagerie électronique étudie principalement le lien utilisation/satisfaction.}

La classe 1 regroupe un vocabulaire spécifique faisant référence aux pratiques de la messagerie électronique et au traitement de l'information dans le Temps. Les items les plus répétés dans les recherches sont en effet :

Reception+(130), traitement+(130), boite+(114), envo + yer(111), jour $+(100)$, responsa $<$, (86), class + er (82), priorite+(78), lettre+(77), tri+(72), arrivee+(69), receptionn +er(66), message $<(62)$, suivre (46), quotidien (45), heure (40)...

Avec 316 U.C.E. cette classe, que l'on peut nommer : "Le temps et la productivité », regroupe deux sous classes :

- La première 1.1 est liée aux différents traitements de l'information : traitement ${ }^{+}$, tri ${ }^{+}$, classer, archive + , priorité+, suivre, copie.

- La deuxième 1.2 est caractérisée par le cadre temporel, on y trouve : fois, jours, arrivée, heure, court, urgent, immédiat, ainsi que les marqueurs d'intensité : assez, beaucoup, chaque...

Les résultats de cette classe nous permettent de dire que le Temps est la variable la plus importante expliquant l'usage des fonctions de la messagerie électronique. Dans cette logique, le Temps est associé à la tâche dans la perspective de productivité déjà citée dans la classe 3 . 


\section{Résultat C : Dans les traitements de l'information effectués par messagerie électronique, c'est le Temps, plus que l'Espace, qui apparaît comme déterminant.}

Enfin, l'analyse thématique de la dernière classe, la classe 2, a révélé les mots suivants :

IHM(365), apprenti<(209), evaluat+ion(192), pedagog<(156), mise-en-œeuvre(145), interac $+(143)$, systeme $+(120)$, variance (115), facteur $+(87)$, artefact $+(80)$, cognitiv $+(77)$

Le pourcentage peu élevé de cette classe (7,2\% soit 148 U.C.E) signifie que peu d'études s'intéressent à l'interface homme/machine. La faible représentativité de cette classe ne nous permet pas de la découper en sous-classes, mais nous permet d'identifier des pistes de réflexion sur la perception de la messagerie. Il est possible de dire que les recherches francophones s'éloignent d'un déterminisme technologique.

En analysant le corpus des recherches, la relation entre la technologie et l'usage est plutôt utilisée dans le cas du système éducatif. Nous nommerons cette classe «Interface homme/machine» et proposerons :

\section{Résultat D : Une minorité de travaux sur la messagerie électronique analyse les interfaces homme/machine, dans une perspective d'apprentissage.}

En résumé nous pouvons donc dire que la quasi-totalité des articles francophones que nous avons analysés sont de type empirique et utilisent la méthode des questionnaires pour répondre aux questions de recherches. Ils traitent de la place de la messagerie électronique par rapport aux autres moyens de communication (téléphone, courrier, face à face...) et des impacts sur la productivité . Enfin, les chercheurs se réfèrent en majorité à la problématique utilisation/satisfaction pour expliquer le choix du média.

\begin{tabular}{|c|c|c|c|}
\hline Classe 3 : Résultat 1 & Classe 4 : Résultat 2 & Classe 1 : Résultat 3 & Classe 2 : Résultat 4 \\
\hline $\begin{array}{l}\text { "Choix des médias et } \\
\text { productivité » } \\
\text { - La productivité associée à } \\
\text { la messagerie électronique } \\
\text { est, dans la plupart des } \\
\text { travaux, liée au problème } \\
\text { du choix des médias } \\
\text { (messagerie, réunions, } \\
\text { écrits, Chat,...). }\end{array}$ & $\begin{array}{l}\text { "Utilisation / } \\
\text { Satisfaction » } \\
\text { - La grande majorité } \\
\text { des travaux sur } \\
\text { la messagerie } \\
\text { électronique étudie } \\
\text { principalement le lien } \\
\text { utilisation/satisfaction. }\end{array}$ & $\begin{array}{l}\text { "Temps et } \\
\text { productivité » } \\
\text { - Dans les traitements } \\
\text { de l'information } \\
\text { effectués par } \\
\text { messagerie } \\
\text { électronique, c'est } \\
\text { le Temps, plus que } \\
\text { l'Espace et le coût, } \\
\text { qui apparaît comme } \\
\text { déterminant. }\end{array}$ & $\begin{array}{l}\text { « Interface homme/ } \\
\text { machine " } \\
\text { - Une minorité } \\
\text { de travaux sur } \\
\text { la messagerie } \\
\text { électronique analyse } \\
\text { les interfaces homme/ } \\
\text { machine dans } \\
\text { une perspective } \\
\text { d'apprentissage. }\end{array}$ \\
\hline
\end{tabular}

Tableau 7 : Récapitulatif des résultats obtenus 


\subsection{Discussion}

Ces résultats nous semblent intéressants, dans la mesure où notre premier objectif était de permettre de caractériser l'orientation de la recherche francophone sur le sujet de la messagerie électronique. Néanmoins trois points peuvent être discutés : sur nos résultats par rapport à une méta-analyse anglophone, sur la validité de notre corpus, et sur les aspects aujourd'hui peu étudiés dans le domaine pour envisager les voies de recherches futures.

Discussion sur les résultats : une comparaison de deux méta-analyses : L'approche de Van den Hooff et al. (2005) va dans le même sens que notre étude, cherchant à connaitre les concepts mobilisés pour étudier l'usage de la messagerie électronique sur un échantillon de travaux anglophones (17 articles). Les chercheurs tentent de regrouper les facteurs influençant l'usage de la messagerie électronique selon quatre grandes variables : L'utilisateur - la tâche l'environnement social - le média.

Les études anglophones sur le sujet étudient alors les relations entre ces quatres variables pour justifier l'utilisation ou la non utilisation du média. L'échantillon sélectionné relève trois types de théories : subsjectives, contingentes et situationelles.

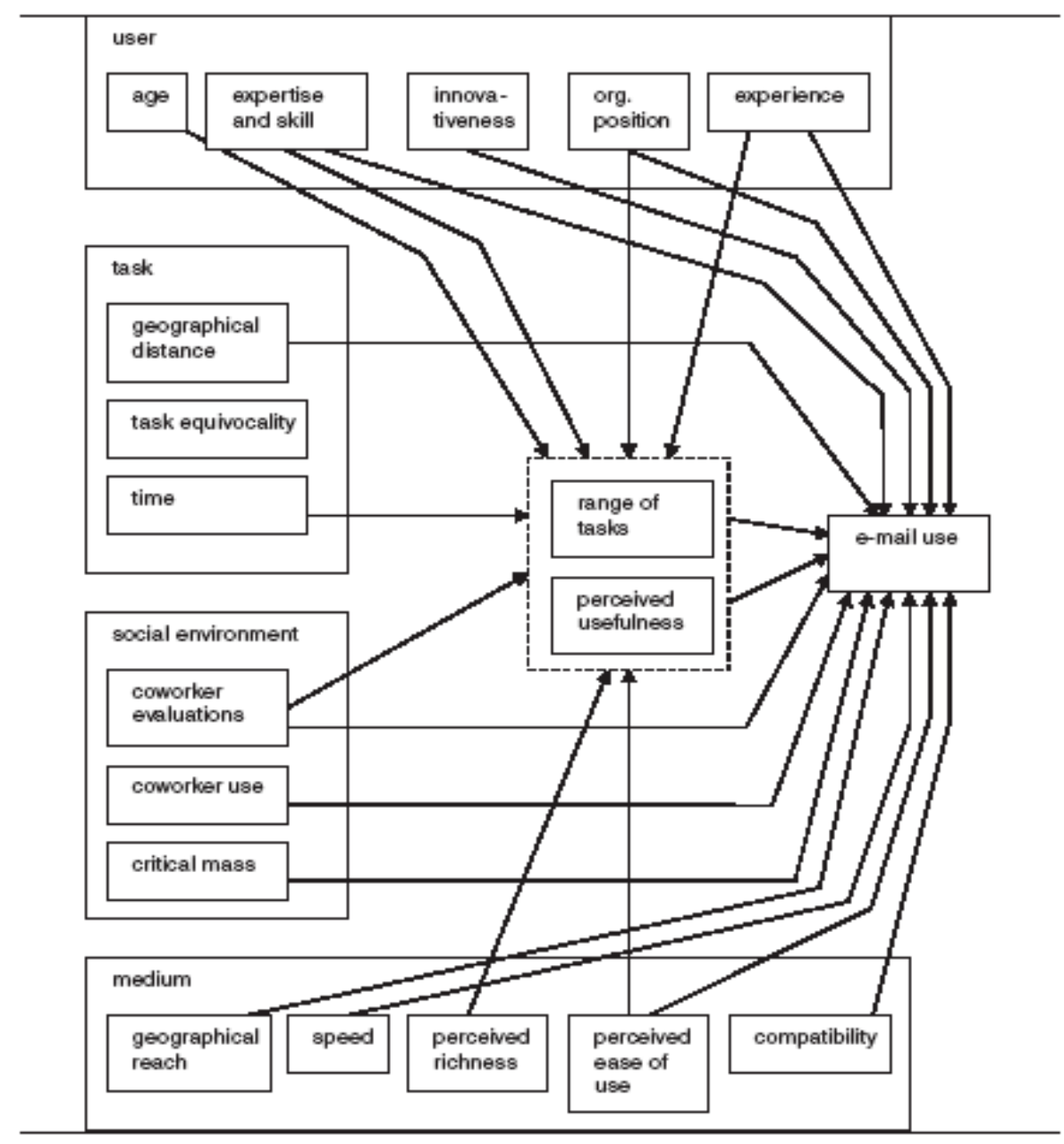


Dès lors, des différences existent entre l'approche de Van den Hooff et al. (2005) et notre approche :

Nous proposons une méthode basée sur une analyse lexicale à travers le logiciel Alceste, différente de celle proposée par Van den Hooff et qui est basée sur une analyse du contenu. Bien que le nombre de travaux sélectionnés soit à peu près comparable (20 recherches francophones, contre 17 anglophones), les objets d'étude ont été plus finement analysés dans la littérature anglo-saxonne.

Les contextes dans lesquels les études respectives ont été menées sont bien sûr différents (anglophones et francophones), ansi que les approches épistémologiques (positivisme, interprétativisme, constructivisme) et les méthodes employées (qualitative, quantitative).

A la différence de la méta-analyse de Van den Hoof, nous n'avons pas isolé de relation entre les variables expliquant l'usage. Sur cette question en effet, notre analyse de type lexical ne permet pas le traitement des ambiguïtés (affirmation ou négation d'une relation), mais ceci constitue pour nous une voie de recherche.

Discussions sur la validité de l'échantillon : Avant d'analyser le corpus nous nous sommes demandé si le nombre d'articles inclus était suffisant pour garantir ces résultats. Dans la démarche de la méta-analyse, l'hétérogénéité est considérée comme un paramètre de nuisance, car elle contredit l'idée d'un traitement commun possible pour toutes les études. Pour cette raison, nous avons préféré privilégier un échantillon de qualité, c'est à dire des travaux ne traitant strictement que de la messagerie électronique. Trois erreurs ou biais sont considérés dans les méta-analyses : erreur d'échantillonnage, biais lié à la différence de fiabilité et biais lié aux différences de dispersion des variables expliquées et explicatives. Le chercheur doit posséder des résultats a priori comparables (Hunter et al. 1982). Or, dans le domaine des systèmes d'information, la logique voudrait que les résultats des études soient convergents et cohérents à la fois dans le sens des relations observées (corrélation positive ou négative) et dans leur intensité (Commeiras et Fournier, 2008). C'est ce que nous avons souhaité examiner dans notre échantillon, qui est certes peu élevé, mais relativement cohérent.

\section{CONCLUSION}

L'objectif de notre contribution était de présenter une méta-analyse dans le domaine des systèmes d'information. A l'aide d'une analyse statistique des données textuelles des travaux de recherches francophones, nous avons analysé

L'usage de la messagerie électronique : une méta-analyse des travaux francophones sur la période 2000-2008

\section{8}

les travaux français qui étudient l'usage de la messagerie électronique dans les organisations sur la période 2000-2008. 
Cette approche nous a permis d'étudier ici un corpus de 20 articles issus de revues scientifiques et de colloques sur la période et d'obtenir quatre classes différentes et quatre résultats associés :

Résultat 1 : La productivité associée à la messagerie électronique est, • pour la plupart des travaux, liée au problème du choix des médias (messagerie, réunions, écrits, Chat,...).

Résultat 2 : La grande majorité des travaux sur la messagerie - électronique étudie principalement le lien utilisation/satisfaction.

Résultat 3 : Dans les traitements de l'information effectués par - messagerie électronique, c'est le Temps, plus que l'Espace et le coût, qui apparaît comme déterminant.

Résultat 4 : Une minorité de travaux sur la messagerie électronique $•$ analyse les interfaces homme/machine dans une perspective d'apprentissage.

Compte tenu des objectifs de notre recherche, nous avons vu l'utilité de réduire le nombre d'articles retenus (biais du résultat final), car la plupart des recherches francophones testent les mêmes variables. Ce travail ne nous permet pas donc de généraliser les résultats et doit se poursuivre dans le cadre d'une méta-analyse anglophone à titre de comparaison.

Aujourd'hui, les modèles " classiques » de type utilisation/satisfaction ont fait l'objet de larges vérifications, notamment dans la littérature anglophone. Il serait donc intéressant que les chercheurs français portent désormais plus d'attention aux liens entre la perception du média et les comportements d'appropriation, notamment dans la perspective de la messagerie sur les postes mobiles et dans une problématique vie privée/ vie professionnelle. Les possibilités offertes par le média peuvent être source d'une «bonne » ou d'une « mauvaise » appropriation (surcharge, contrôle, ambiguïté, conflit...).

\section{BIBLIOGRAPHIE}

Autissier D. (2000), «Utilisation de la messagerie électronique dans une multinationale : l'émergence d'un nouveau média », Colloque Sciences de l'Information et Gestion, Nice, 17 et 18 Décembre.

Bachelet C. et Caron Fasan ML. (2000), «Introduction de la messagerie et des forums dans l'entreprise, quels impacts pour l'organisation ? Une approche exploratoire», 5éme Colloque de l'AIM, Montpellier, 8-10 Novembre.

Bachelet C. et Moscarola J. (2001), « La messagerie électronique, facteur de changement dans l'organisation ? Implication sur la décision », 5éme Colloque du CRIC, Nice, 6 et 7 Décembre.

Bachelet C. et Moscarola J. (2002) « La messagerie électronique dans la communication : Usages, Contextes et satisfaction », 7éme Colloque de l'AIM, Hammamet, Tunisie, 30 Mai-1 Juin.

Baile S. (2004), « Pourquoi et Comment évaluer l'Interaction Homme-Machine ? Le cas de la messagerie électronique dans les processus d'apprentissage», 9ème Colloque de l'AIM, Evry, 26-28 mai. 
Baile S. et Lefievre V. (2003), «Le succès de l'utilisation de la messagerie électronique - étude de ses déterminants au sein d'une unité de production aéronautique », 8ème Colloque de l'AIM, Grenoble, 21-23 mai.

Barry P. et Diop H. (2002), «L'impact d'Internet sur le fonctionnement des moyennes et grandes entreprises industrielles », Le Sénégal à l'heure de l'information, Karthala-UNRISD, Paris, pp. 97-120.

Baumard P. et Ibert J. (1999), Chapitre IX : La gestion des sources de données, in Méthodologie de la recherche en gestion, édition R.A. Thiétart, Paris.

Boukef N. et Kalika M. (2002), « Facteurs déterminants de l'utilisation du courrier électronique », $7^{\text {éme }}$ Colloque de l'AIM, Hammamet, Tunisie, 30mai - 1juin.

Boukef Charki N. et Charki M.H. (2008), «L'e-mail : un moyen de contrôle ou de responsabilité ?», Systèmes d'Information et Management, Vol. 13, $\mathrm{n}^{\circ} 4$.

Commeiras N. et Fournier C. (2008), «Comment générer des pistes de recherche à partir des variables modératrices mises en évidence lors d'une méta-analyse? Une illustration », Congrès du réseau des IAE, Lille - 10, 11 et 12 Septembre.

Cucchi C. (2004), « Représentation graphique de la communication organisationnelle par les réseaux sociaux; exemple des échanges électroniques », 13éme Colloque de l'AIMS, Vallé de Seine, 2-4 juin.

Cucchi A. et Cucchi C. (2003), « Complémentarité et substitution du téléphone et du courrier électronique : une approche par typologie prédictive », $12^{\text {éme }}$ colloque de l'AIMS, Tunisie, 3-6 Juin.

Cudicio R. et Proulx S. (2006), « La messagerie instantanée en entreprise : Accélérateur ou frein à la productivité ? », Colloque semaine de la connaissance, Nantes, 26-30 juin.

Daft R.L. et Lengel R.H. (1986), “Organizational Information Requirements, Media Richness and Structural Design”, Management Science, Vol. 32, n5, pp. 554-571.

Daoud H., Hassairi A.F et Triki M. (2007), « Influence de l'utilité perçue et de la facilité d'usage sur l'utilisation de la messagerie électronique: application au contexte tunisien », Colloque Internationale de l'Ecommerce et Gouvernance de l'Internet, Sousse, Tunisie, 19 et 20 octobre.

Davis F.D. (1989), "Perceived usefulness, perceived ease of use, and user acceptance of information technology", MIS Quarterly, Vol 13, n³, pp. 319-340.

DeLone W.H. et McLean E.R. (2003), "The DeLone and McLean model of Information Systems Success", Journal of Management Information Systems; $\mathrm{n}^{\circ} 19$, pp. 9-30. 
DeSanctis G. et Poole M.S. (1994), "Capturing the complexity in advanced technology use: Adaptive Structuration Theory”, Organization Science, Vol. 5, n², pp 121-147.

De Rosnay J. (1975), Le Macroscope : vers une vision globale, Seuil Edition, Paris, 346 p.

De Vaujany F.X., (2000), «Usages d'un intranet et processus de structuration de l'organisation», Systèmes d'Information et Management, Vol.5, n², pp. 79-100.

D’Iribarne A. et Gadille M. (2000), « La diffusion d'Internet dans les PME : motifs d'adoption, réseaux et ressources mobilisées », Réseaux, n¹04, pp. 61-92.

Eisenhardt K.M. (1989), "Building theories from case study research", Academy of management review, Vol. 14, n532, pp. 57-74.

Fallery B., Rodhain F. (2007), «Quatre approches pour l'analyse de données textuelles : lexicale, linguistique, cognitive, thématique », 14éme conférence Internationale de Management Stratégique (AIMS), Montréal, 7-9juin.

Giddens, A. (1987), La constitution de la société, PUF Editeur, Paris, 474p.

Glass G.V. (1976), "Primary, secondary and meta-analysis of research”. Educational Researcher, n 10 , pp. 3-8.

Gleonnec M. (2004), «Messagerie électronique synchrone et structuration du lien social en entreprise », Colloque Organisation Media, Lyon, 19 et 20 novembre.

Guilloux V., Gauzente C. et Kalika M. (2000), « Grandeurs et limites de la communication électronique : analyse d'un cas de projet de recherche marketing », CREPA, Cahier de recherche, n56, pp.1-18.

Hunter J.E., Schmidt F.L. et Jackson G.B. (1982), Meta-analysis: Cumulating research findings across studies, Sage Publications, Beverly Hills, CA.

Jackson T.W., Dawson R. et Wilson D. (2003), "Reducing the effect of e-mail interruptions on employees", International journal of Information management, Vol. 23, $\mathrm{n}^{\circ} 1, \mathrm{pp}$. 55-65.

Jouët J. (2000), Retour critique sur la sociologie des usages, Réseaux, n¹00, Vol. 18, pp 487-521.

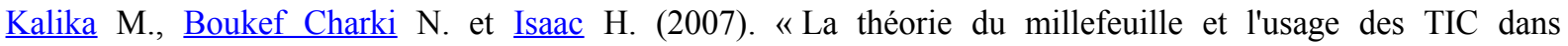
l'entreprise », Revue Française de Gestion, Paris. Vol. 33, n 172, pp. 117-129. 
Labbe H. et Marcoccia M. (2005), « Communication numérique et continuité des genres : l'exemple du courrier électronique », Version revue de «Tradition épistolaire et médias numériques : du billet au courrier électronique ». In A. Betten \& M. Dannerer (eds.), vol. 2, pp. 281-292.

Le moigne J.L. (1990), La modélisation des systèmes complexes, Afcet système, Dunod, Paris.

Limayem M., Bergeron F., Richard A. (1997), « Utilisation des messageries électroniques: mesures objectives versus mesures subjectives », Systèmes d'Information et Management, n¹, vol. 2, pp 51-69.

Mcluhan M. (1964), Understanding Media, The extension of man, NY, McGraw-Hill.

Moscarola J. (2001), «Contributions des méthodes de l'analyse qualitative à la recherche en psychologie interculturelle : Sphinx et MCA », Sème Congrès International de l'ARIC, Genève, 24-28 Septembre.

Orlikowski W.J. et J.J. Baroudi (1991), "Studying Information Technology in Organizations: Research Approaches and Assumptions”. Information Systems Research Vol. 2, n¹, pp. 1-28.

Orlikowski W.J (2003), “'L'art du management de l'information”,

« http://www.lesechos.fr/formations/manag_info/articles/article 8 5.htm $»$.

Raymond L. (2002), "L'impact des systèmes d'information sur la performance de l'entreprise », in Faire de la recherche en système d'information, ouvrage coordonnée par F. Rowe, op. cit., Paris, pp. 301-320.

Raymond L. et Bergeron F. (1996), "EDI Success in Small and Medium-Sized Enterprises: A Field Study", Journal of Organization Computing and Electronic Commerce, Vol. 6, n² 2, pp. 161-172.

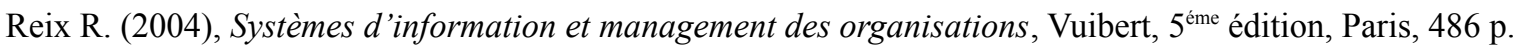

Rolland S. et Tran S. (2007), « L'impact des TIC sur la productivité : la dimension oubliée de la structuration des usages ? Le cas de l'email dans les secteurs de services », Colloque ECIG, Sousse, Tunisie, 19 et 20 octobre.

Rowe F. et Monod E. (2000), « Limites structurels et culturelles à l'usage de la messagerie électronique dans les banques », Réseaux, Vol 18, n¹04.

Saintive B. (2000), «Trois formes différenciées d'usage de la messagerie électronique au sein d'une organisation », Réseaux, n¹04, vol. 18, pp. 119-136.

Simon H.A. (1991), “Cognitive architectures and rational analysis” Comment in Vanlehn K. (Ed.), Architectures for intelligence, Hillsdale, pp. 25- 39. 
Tahri W. (2008), « La messagerie électronique dans les multinationales : sources de productivité ou de conflits?

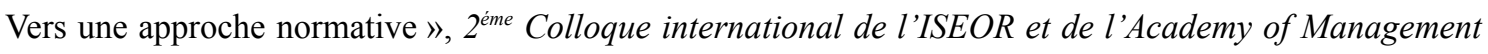
(USA), Lyon, 10 et 11 mai.

Van den Hooff B., Groot J. et de Jorge S. (2005), "Situational influences on the use of communication technologies: A meta analysis and exploratory study”, Journal of business Communication, Vol. 41, n², pp. 4-27.

Wacheux F. (1996), Méthodes qualitatives et recherche en gestion, Economica, Paris, 290p.

Watzlawick P., Helmick-Beavin J. et Jackson D.D. (1972), Une logique de la communication, Edition du Seuil, Paris.

Weber R. (2004), “The grim reaper: the curse of e-mail”, MIS quarterly, Vol. 28, n³, pp. 3-14.

Webster J. et Trevino L.K. (1995), "Rational and social theories as complementary explanations of communication media choices: two policy-capturing studies", Academy of management journal, Vol. 38, $\mathrm{n}^{\circ} 6$, pp. $1544-1572$

Weick K.E. (1995), Sensemaking in organizations, Sage publications, California, 231p.

Yousafzai S.H., Foxall G.R. et Pallister J.G., "Technology acceptance: a meta-analysis of the TAM: Part1", Journal of Modelling in Management, Vol. 2, n³, pp. 251-280.

Yin Robert K. (2003), Case study research, design and methods, 3rd edition, Sage publications, London, 181p. 